\title{
Communication
}

\section{Premier isolement au Tchad du virus de la PPR et reproduction expérimentale de la maladie}

\author{
K. Bidjeh ${ }^{1}$ \\ P. Bornarel ${ }^{1}$ \\ M. Imadine ${ }^{1}$ \\ R. Lancelot ${ }^{1 *}$
}

BIDJEH K., BORNAREL P., IMADINE M., LANCELOT R. Premier isolement au Tchad du virus de la PPR et reproduction expérimentale de la maladic. Revue Elev. Méd. vét. Pays trop., J995, 48 (4) : 295-300

Des foyers de la peste des petils runiniluts (PPR) ont été étudiés en 1993 et 1994 dans des troupeaux de chèvres de race sahélienne au Tchad. Si le virus n'a pu être isolé des premiers foyers de 1993, le sondage sérologique par le test ELISA de compétition portant sur 475 sćrums récoltés dans la zone d'épidémie a montré une prévalence de $34 \mathrm{p}$. 100. Pour la première fois au Tchad, le virus fut isolé et des cas cliniques caractéristiques de la PPR furent observés sur les chèvres sahéliennes réputées peu sensibles à ce virus. La maladie a été reproduite expérimentalement par inoculation des chèvres avec des broyats de ganglions et de poumons, ces derniers ayant provoqué les symptômes les plus sévères. La réaction d'immunodiffusion en gélose s'est révélée positive vis-à-vis de l'antisérum anti-PPR avec les broyats de poumons et de ganglions mésentériques, mais pas avec ceux de ganglions préscapulaires. La caractérisation du virus isolé, en particulier sur le plan génomique, s'avère nécessaire.

Mots-clés : Bovin - Caprin - Morbillivirus - Infection expérimentale Sérologie - Test ELISA - Immunologie - Epidémiologie - Tchad.

\section{Introduction}

La peste des petits ruminants (PPR) est une maladie largement répandue en Afrique et en Asie (Moyen Orient et Inde). Cette pathologie, proche de celle de la peste bovi-

1. Laboratoire de Recherches vétérinaires et zootechniques de Farcha, BP 433, N'Djaména, Tchad.

*. Adresse actuelle : ISRA/LNERV, BP 2057, Dakar Hann, Sénégal.

Reçu le 28.2.95, accepté le 23.2.96. ne au plan clinique, constitue un frein pour l'épanouissement de l'élevage des petits ruminants car les pertes économiques qu'elle entraîne sont considérables. L'agent pathogène responsable est un virus appartenant au genre Morbillivirus, famille des Paramyxoviridæ (8). Pendant longtemps, ce virus fut considéré comme un mutant du virus de la peste bovine adapté aux petits ruminants et ayant perdu tout pouvoir pathogène pour les bovins $(4,13)$. Ce n'est qu'après des études approfondies que certains chercheurs $(3,5,8,9)$ ont affirmé qu'il s'agissait de deux entités distinctes. Bien que tous les pays limitrophes, la Libye exceptée, aient déclaré la présence de la PPR, au Tchad son existence n'a encore été révélée que par des enquêtes sérologiques $(2,14)$. Le présent article décrit des foyers de PPR dans plusieurs troupeaux de la zone périurbaine de N'Djaména et dans une localité située à $150 \mathrm{~km}$ au sud de la capitale (N'Djénéré) ainsi que l'isolement du virus, son identification et la reproduction expérimentale de la maladie sur des sujets sensibles.

\section{Matériel et Méthodes}

Les animaux utilisés pour l'expérimentation sont un bouvillon et huit chèvres toutes de race kirdimi reconnue hautement sensible au virus de la PPR (10). Tous les animaux sélectionnés sont séronégatifs vis-à-vis des virus de la PPR et de la peste bovine. Ils sont logés dans une étable composée de quatre boxes cloisonnés par un mur surmonté d'un grillage, de façon à empêcher les animaux de se toucher. Trois compartiments abritent chacun deux chèvres. Le quatrième contient le bouvillon et les deux autres chèvres. Les sujets des trois premiers lots reçoivent par voie sous-cutanée $4 \mathrm{ml}$ du broyat des poumons, des ganglions préscapulaires et mésentériques, respectivement. Ces prélèvements proviennent d'un animal malade infecté naturellement. Ceux du quatrième lot reçoivent la même dose mais du broyat de ganglions préscapulaires d'un des deux sujets morts à la suite de l'infection expérimentale.

A la fin des expériences ( 30 jours), les sérums sont récoltés sur les animaux survivants et ceux n'ayant pas réagi à l'inoculation du virus, pour la recherche des anticorps anti-PPR qui sont titrés par le test de séroneutralisation décrit par Taylor (16). Le virus utilisé pour ce test est celui de la souche vaccinale PPR 75/1 LK6 vero 67 (6). 
Ceux récoltés sur le terrain sont traités qualitativement au CIRAD-EMVT par la méthode ELISA de compétition mise au point par Libeau ct coll. (12). Par cotte méthode, un sérum est considéré positif si le pourcentage de compétition est supérieure à 50 p. 100.

L'isolement du virus à partir des broyats des organes est fait sur des cellules de rein de mouton au deuxième passage. Le milieu de culture est constitué de MBE (Laboratoire Eurobio, France) additionné d'acides aminés, de vitamines, de sérum de veau fœtal (10 p. 100), de pénicilline $(100 \mu \mathrm{g} / \mathrm{ml})$ et de streptomycine $(0,05 \mathrm{mg} / \mathrm{ml})$. Une fois que le tapis cellulaire est complet dans la boîte de culture, le milieu est remplacé par un autre contenant $1 \mathrm{p}$. 100 de sérum (milieu d'entretien).

Les organes prélevés sur les animaux morts sont aussi testés par la réaction d'immunodiffusion dans un milieu gélifié (IDG) avec un antisérum anti-PPR gracieusement offert par le Dr Diallo (CIRAD-EMVT, France). La mise en évidence des antigènes PPR par la méthode d'immunocapture (11) est réalisée au CIRAD-EMVT.

\section{Résultats}

\section{Dans les foyers de la maladie}

En mars 1993, 15 cas de PPR dans sept troupeaux appartenant à cinq villages différents sont signalés dans le troupeau de Djékémé, village situé sur l'axe N'Djaména- Djermaya. Les animaux atteints sont surtout des chevreaux de race sahélienne, âgés de 3 à 6 mois. On observe les signes cliniques suivants : jetage, toux, larmoiement, diarrhée profuse et profonde atteinte de l'état général (figure 1). Certains sujets présentent des lésions de la cavité buccale : ulcères, liseré congestif à la base

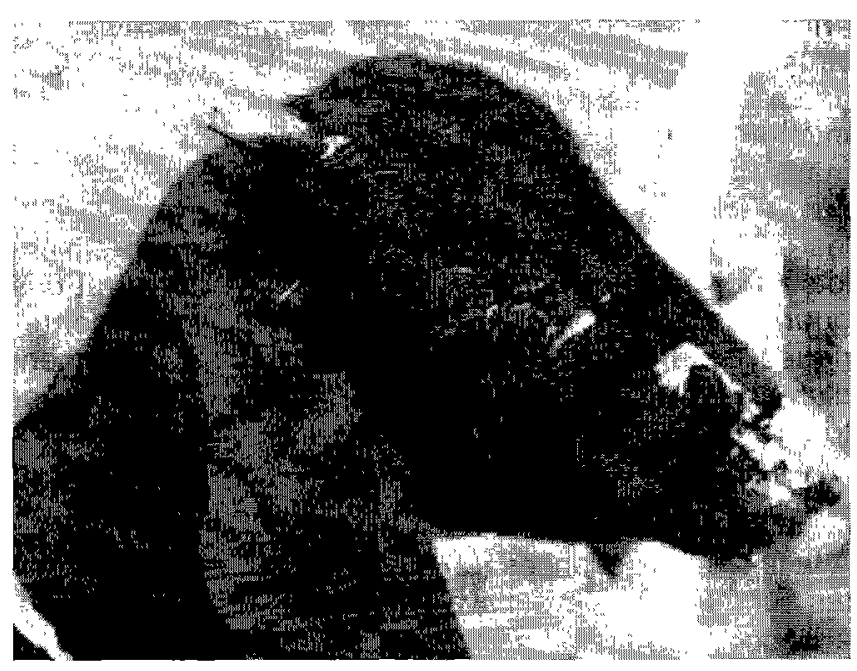

Figure 1 : Jetage mucopurulent et larmoiement. des gencives, érosions recouvertes d'un enduit pultacé blanchâtre saignant au moindre contact (figures 2 et 3 ). Des cas do mortalitć sont enregistrés. Dans l'un des élevages atteints, les prélèvements suivants sont effectués sur des animaux malades: frottis des larmes, des muqueuses buccale et nasale, sang sur anticoagulant. Un chevreau est sacrifié et à l'autopsie nous observons une congestion du mésentère et des ganglions environnants, des foyers d'hépatisation rouge des lobes apicaux des poumons.

Les tentatives d'isolement du virus et de reproduction expérimentale de la maladie sur des animaux sensibles se sont soldées par un échec. En revanche, la recherche d'antigènes dans les frottis lacrymaux par la méthode

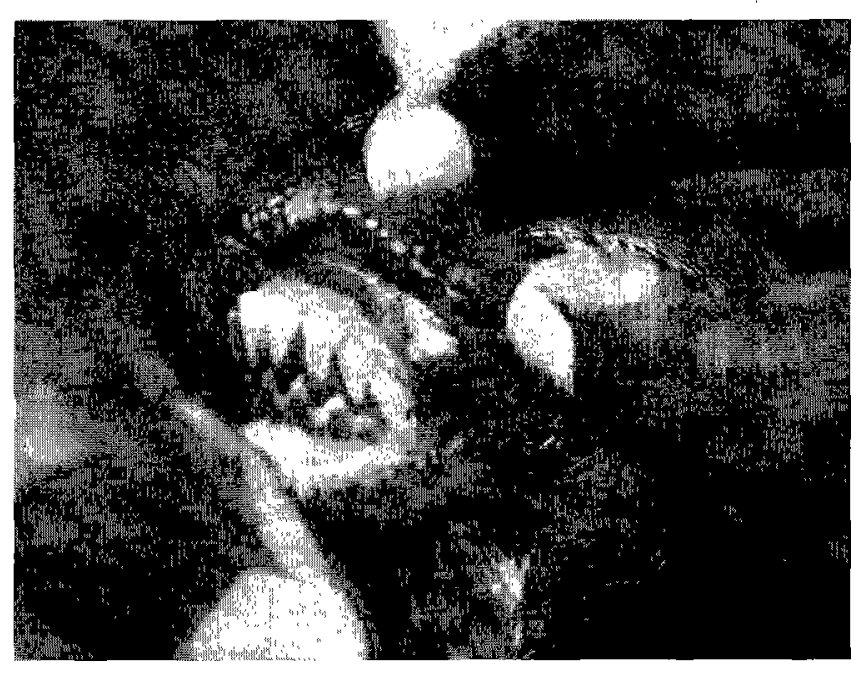

Figure 2 : Lésions buccales : enduit pultacé blanchâtre.

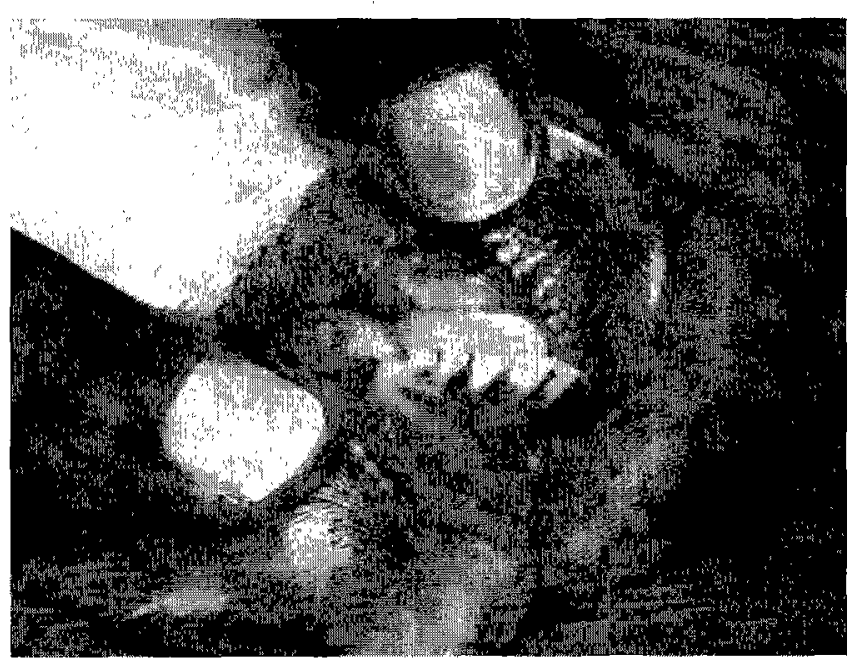

Figure 3 : Lésions buccales : érosion. 
d'immunocapture s'est avérée positive sur un des échantillons. Les résultats sérologiques effectués sur 475 échantillons par le test ELISA de compétition PPR ont montré 33,7 p. 100 des sérums positifs et 3,8 p. 100 douteux.

En avril 1994, à Djénéré, localité située à $150 \mathrm{~km}$ au sud de N'Djaména, dans un troupeau de 36 chèvres de race sahélienne, il y a une morbidité de 85 p. 100 et une mortalité de 47 p. 100 . Les animaux malades présentent les signes suivants : hyperthermie $\left(41,3^{\circ} \mathrm{C}\right.$ en moyenne), larmoiement, jetage abondant et mucopurulent (figures 1, 4, 5), dyspnée, diarrhée profuse et lésions buccales. Un des animaux malades est sacrifié sur place.

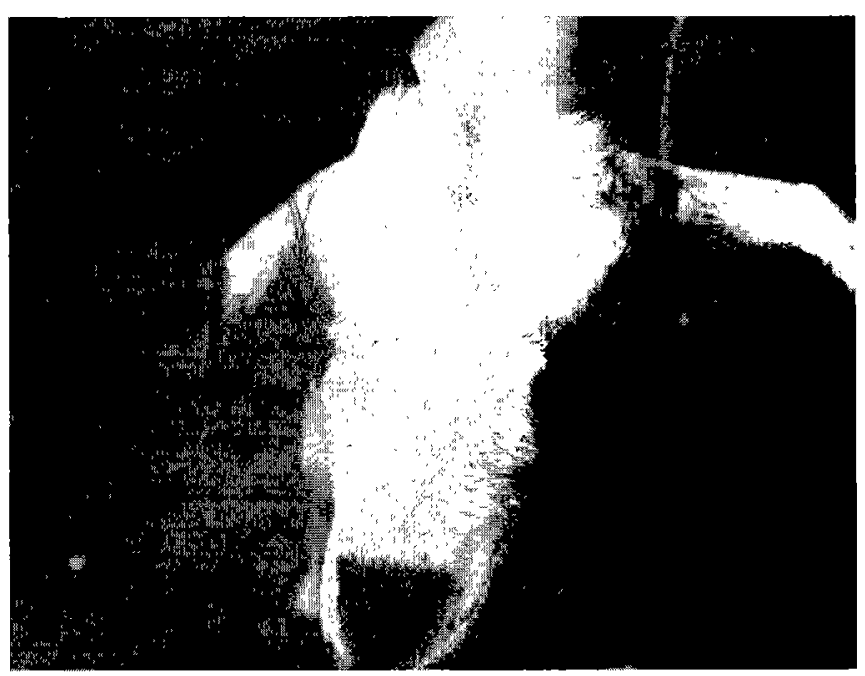

Figure 4 : Larmoiement bilatéral.

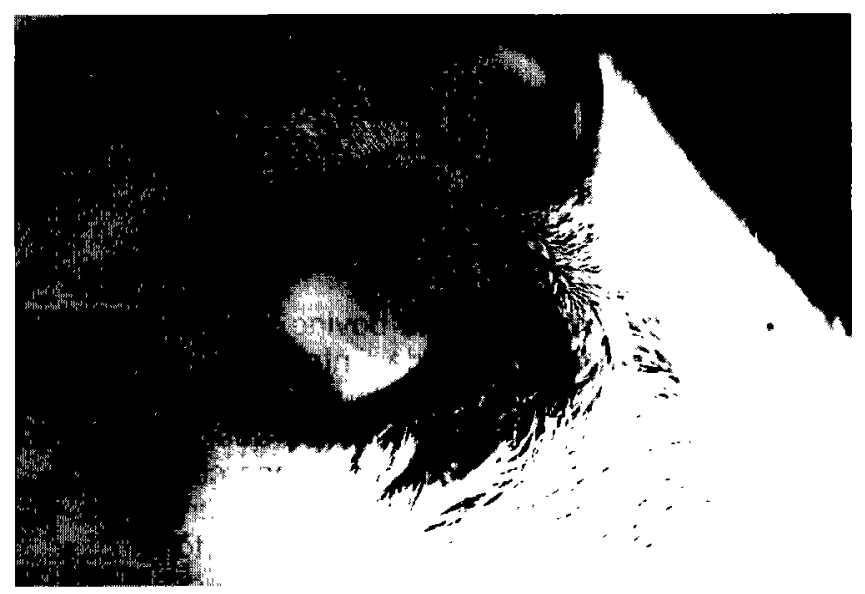

Figure 5 : Congestion de la conjonctive.
A l'autopsie, on retrouve les mêmes lésions décrites lors du foyer de 1993. Des ganglions, des lésions de la langue, des poumons et du cæcum sont prélevés (figure 6). Dans le troupeau, certains animaux avaient des signes cliniques moins prononcés (figure 7). Les moutons ne semblent pas être atteints de mème que les autres espèces présentes (bovins, camelins, équins et asins). Selon les éleveurs, la maladie serait apparue dans le village pour la première fois en 1993, probablement après contact avec des troupeaux transhumants venant s'abreuver au puits du village.

En juillet 1994, c'est-à-dire trois mois plus tard, les auteurs retournent sur les lieux et constatent qu'un seul chevreau présente les signes cliniques relatifs à la PPR (exceptées les lésions buccales). Onze sérums sont col-

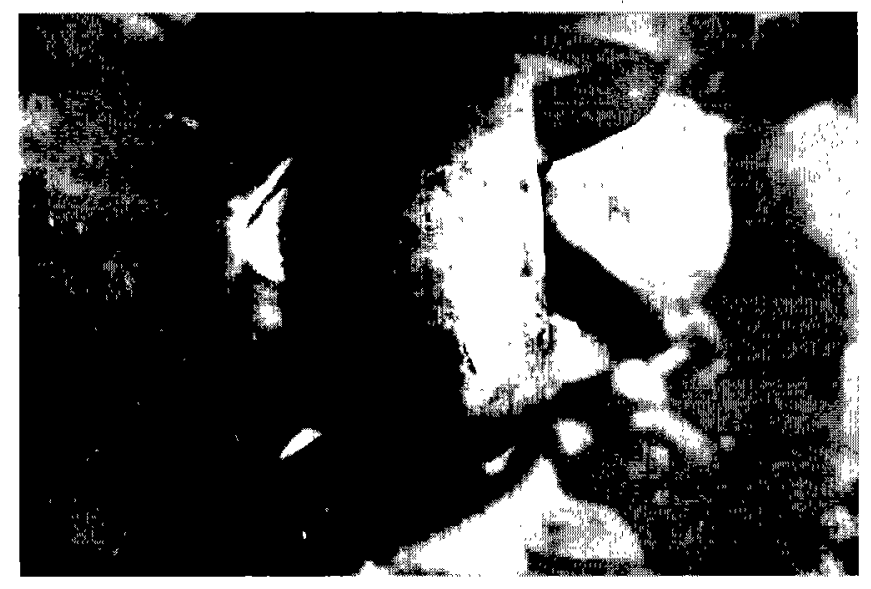

Figure 6: Pétéchies sur le cacum.

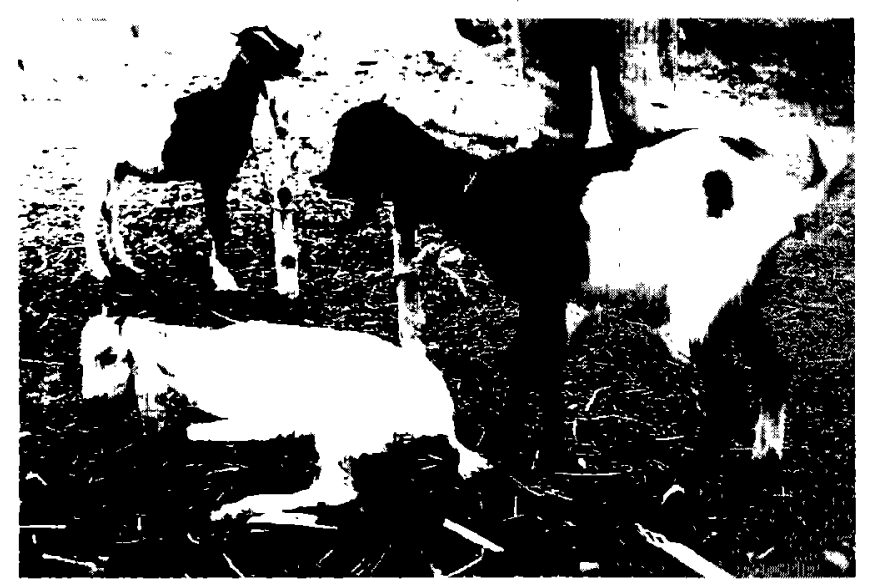

Figure 7 : Animalx atteints de PPR dans un troupeau. 
TABLEAU I

Reproduction expérimentale de la PPR chez les chèvres kirdimi tchadiennes à partir d'organes d'un animal malade, infecté naturellement, sacrifié
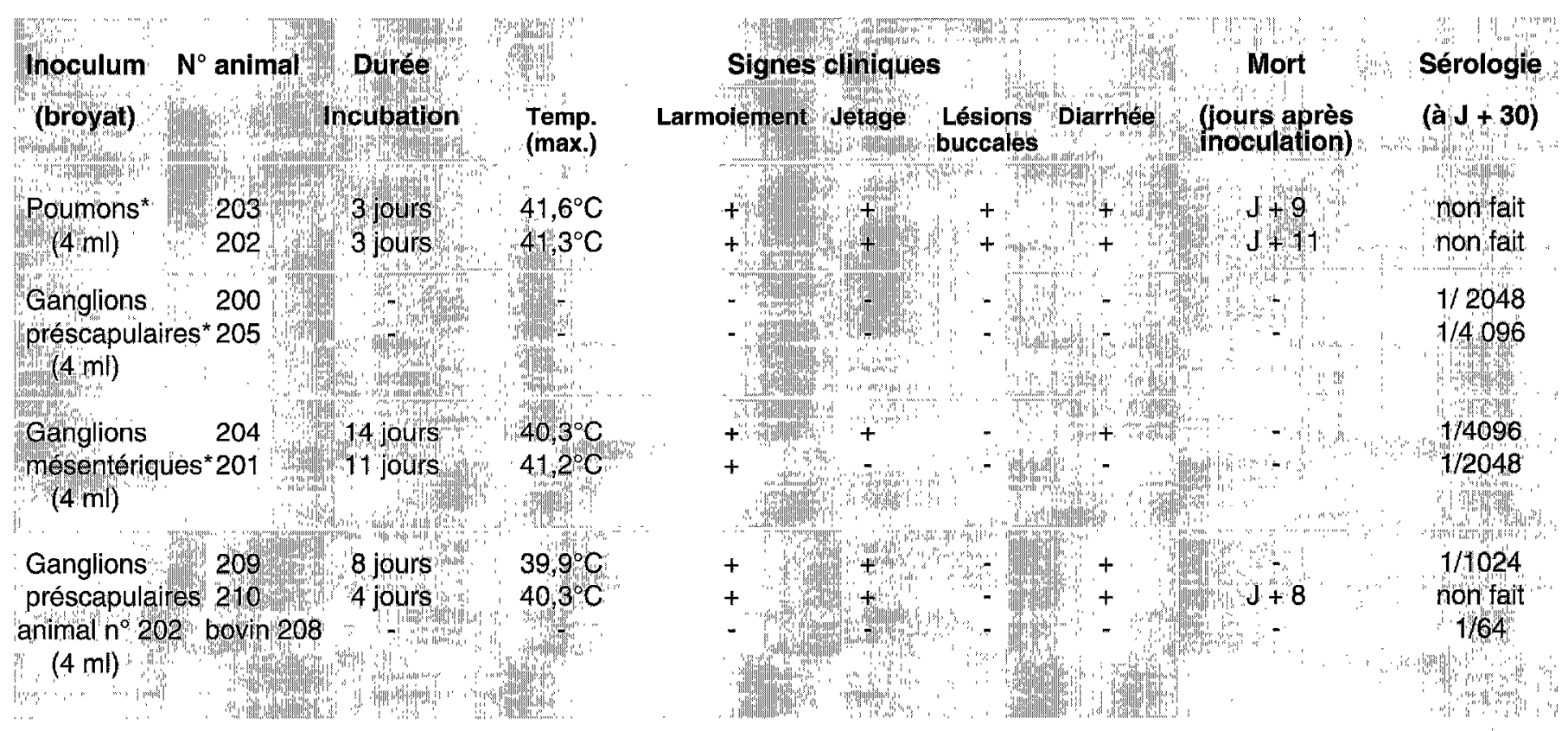

* Provenant de l'animal malade issu du foyer naturel

lectés au hasard sur les animaux et l'analyse sérologique par le test de séroneutralisation montre que tous hébergent des anticorps anti-PPR à des titres variant entre $1 / 32$ et $1 / 516$.

\section{Reproduction expérimentale de la maladie}

Les broyats des organes (poumons, ganglions mésentériques et préscapulaires), prélevés de l'animal sacrifié lors de l'épidémie d'avril 1994, sont inoculés à des chèvres sensibles. Du tableau I, on peut remarquer que tous les animaux qui ont reçu le broyat de poumons sont morts au $9^{\circ}$ et $11^{\circ}$ jour après avoir présenté tous les signes cliniques de la maladie. Aucun signe clinique relatif à la PPR n'a été observé chez les sujets ayant reçu le broyat des ganglions préscapulaires (figure 8 et tableau I). En revanche, ces animaux ont fait une séroconversion au $30^{e}$ jour après l'inoculation (titre de 1/2 048 et $1 / 4096$ en séroneutralisation). Tous les signes cliniques ne sont pas observés chez les chèvres qui ont reçu le broyat de ganglions mésentériques (tableau l) : ainsi, les lésions buccales sont absentes, le jetage et la diarrhée sont remarqués chez l'un des deux sujets ; aucune mortalité n'est enregistrée dans ce groupe. Les deux chèvres survivantes ont fait une séroconversion (titre de $1 / 2048$ et $1 / 4096$ ) comme dans le cas précédent. Chez tous les animaux ayant réagi positivement à l'inoculation de différents organes, on constate une

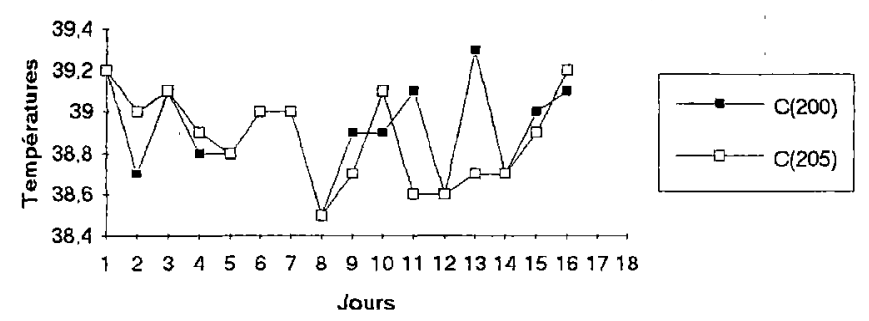

Figure 8: Courbes thermiques - Inoculation à partir de broyat de ganglion préscapulaire.

hyperthermie variant entre $39,9^{\circ}$ et $41,6^{\circ} \mathrm{C}$ (figures 9 et 10), hyperthermie qui amorce toujours le début de la maladie. La durée d'incubation a été de 3 à 11 jours suivant le cas.

Dans le but d'exclure la peste bovine, le broyat de ganglions d'un des sujets mort de l'infection expérimentale est inoculé à un bouvillon et à deux chèvres. Comme on peut le constater sur le tableau I, les deux chèvres ont réagi à l'inoculation avec tous les signes cliniques de la PPR (exceptées les lésions buccales). L'une d'elle est morte le $8^{\circ}$ jour après infection. Aucun symptôme clinique relatif à la PPR ni à la peste bovine n'est observé chez le bouvillon (figure 11). Le bouvillon et la chèvre qui a survécu à l'infection expérimentale ont fait une séroconver- 


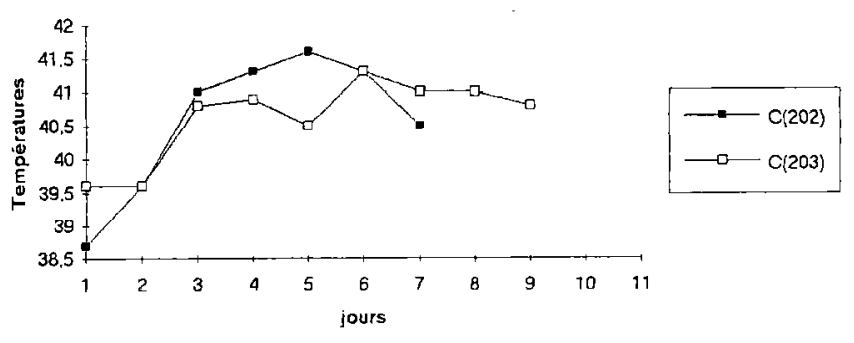

Figure 9: Courbes thermiques - Inoculation à partir de broyat de poumon.

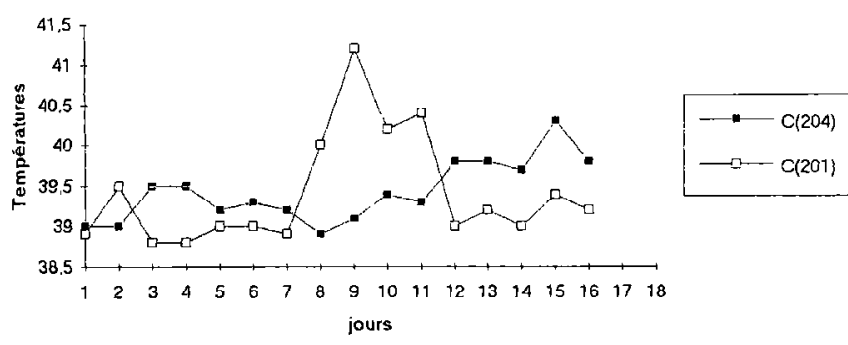

Figure 10: Courbes thermiques - Inoculation à partir de broyat de ganglion mésentérique.

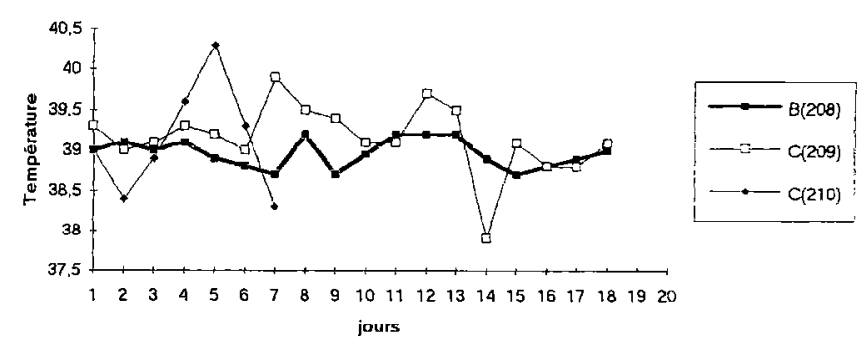

Figure 11: Courbes thermiques - Inoculation à partir de ganglion préscapulaire de la chèvre 202.

sion avec des titres d'anticorps anti-PPR respectivement de $1 / 64$ et $1 / 1$ 024. Par ailleurs, la réaction d'immunodiffusion en gélose s'est révélée positive vis-à-vis de l'antisérum anti-PPR avec les broyats de poumons et de ganglions mésentériques, mais pas avec les ganglions préscapulaires. A partir des broyats de poumons, un virus a été isolé sur les cellules de rein de mouton en culture. L'effet cytopathogène a été constaté à partir du $7^{e}$ jour après inoculation. II se caractérise par l'apparition de cellules multinuclées avec une couronne des noyaux réfringents et une masse cytoplasmique amorphe.

\section{Discussion et Conclusion}

Pour la première fois au Tchad, des cas cliniques caractéristiques de la PPR sont observés sur les chèvres sahéliennes réputées peu sensibles à ce virus (10). Toutefois l'expression de la maladie a été peu fréquente et ne s'est révélée grave voire alarmante que dans deux élevages.

Les symptômes observés sur le terrain chez ces chèvres sahéliennes pendant les deux dernières années sont similaires à ceux déjà décrits par différents auteurs, dont Gargadennec en 1942 (7), Rowland en 1970 (15) et Abu el Zein en 1990 (1).

A partir de broyats d'organes prélevés d'un animal sacrifié au cours d'une épidémie, la maladie a été reproduite expérimentalement par inoculation des chèvres kirdimi, bien que ces dernières, chèvres naines, soient réputées très sensibles à la PPR (10). Tous les signes cliniques évoqués ci-dessus n'ont pas été exprimés par ces animaux hormis deux cas. Ainsi, le jetage, la diarrhée et les lésions érosives de la muqueuse buccale ont été les symptômes les plus inconstants chez les sujets ayant reçu les broyats de ganglions. Aucune mortalité n'a été notée avec les ganglions mésentériques et préscapulaires. Cependant les titres d'anticorps anti-PPR sont très élevés après 30 jours d'observation. En revanche, les animaux inoculés avec le broyat de poumon ont fait une forme sévère de la maladie et sont morts.

La réaction positive des broyats des organes avec l'antisérum anti-PPR en immunodiffusion, la réussite de la reproduction expérimentale de la maladie chez des chèvres avec une forte séroconversion chez les survivants, l'absence de réaction du bouvillon à l'inoculation du matériel infectieux sont des résultats qui plaident en faveur de la présence de la peste des petits ruminants. Pour la première fois au Tchad, les auteurs ont réussi à isoler le virus en cause. Sa caractérisation, en particulier sur le plan génomique, s'avère maintenant nécessaire.

\section{Bibliographie}

1. ABU EL ZEIN E.M.. HASSANIEN M.M., AL AFALEQ A.I., HOUSSAWI F.M., 1990. Isolation of "peste des petits ruminants" from goats in Saudi Arabia. Vet. Rec., 127 : 309-310.

2. BIDJEH K., IDRISS A.O., DIGULMBAYE C., GANDA K., MAURICE Y., 1989. Incidence des anticorps peste bovine chez les petits ruminants au Tchad. Bull. Cent. Rech. appl. (CRA). 5 : 31-36.

3. BARRETT T.. UNDERWOOD B., 1985. Comparison of messager RNAs induced in cells infected with each member of morbillivirus group. Virology, 145: 195-199.

4. BOURDIN P., RIOCHE M., LAURENT A., 1970. Emploi d'un vaccin antibovipcstique produit sur cultures cellulaires dans la prophylaxie de la peste des petits ruminants au Dahomey. Revue Elev. Méd. vét. Pays trop., 23 (3) : 295-300 


\section{Communication}

5. DIALLO A., BARRETT T., LEFEVRE P.-C., TAYLOR W.P., 1987 Comparison of proteins induced in cells infected with rinderpest and PPR viruses. J. gen. Virol., 68: 2033-2038.

6. DIALLO A., TAYLOR W.P., LEFEVRE P.-C., PROVOST A., 1989. Atténuation d'une souche de virus de la peste des petits ruminants : candidal pour un vaccin homolugue vivantl. Revue Elev. Méd. vét. Pays trop., 42 (3) : 311-319.

7. GARGADENNEC I., LALANNE A., 1942-1943. La peste des petits ruminants. Bull. Serv. Epizoot. AOF, 5-6 : 6-21.

8. GIBBS E.C.J., TAYLOR W.P., LAWMAN M.J.P., BRYANT J, 1979. Classification of "peste des petits ruminants" virus as the fourth member of genus Morbillivirus. Intervirology, 11: 268-274.

9. HAMDY F.M., DARDIRI A.H., BREESE S.S. Jr., DEBOER C.J., 1976. Immunological relationship between rinderpest and "peste des petits ruminants" viruses. Proc. 79th Meet. U.S. Anim. Health Assoc.: 168-179.

10. IDRISS A.O., BIDJEH K., GANDA K., DIGUIMBAYE C., MAURICE Y., 1989. Sensibilité des races ovines et caprines tchadiennes au virus de la PPR. In: Wilson R.T. et Azeb M. eds, African small ruminant Research and Development. Addis Ababa, Ethiopia, ILCA, p. 370-381.

11. LIBEAU G., DIALLO A., COLAS F., GUERRE L., 1994. Rapid differencial diagnosis of rinderpest and "peste des petits ruminants" using an immunocapture ELISA. Vet. Rec., 134: 300-304.

12. LIBEAU G., PREHAUD C., LANCELOT R., COLAS F., GUERRE L., BISHOP D.H.L., DIALLO A., 1995. Development of a competitive ELISA for detecting antibodies to the "peste des petits ruminants" virus using a recombinant Nucleoprotein. Res. vet. Sci., 58: 50-55.

13. MORNET P., ORUE J., GILBERT Y., THIERRY G., SOW M., 1956. La peste des petits ruminants en Afrique occidentale française. Ses rapports avec la peste bovine. Revue Elev. Méd. vét. Pays trop., 9 ; 313-342.
14. PROVOST A., 1973. Pcstc des petits ruminants. Farcha, Tchad, Laboratoire de Recherches vétérinaires et zootechniques de Farcha, p. 106-108. (Rapport annuel)

15. ROWLAND A.C., BOURDIN P., 1970. The histological relationship between "peste des petits ruminants" and kata in West Africa. Revue Elev. Méd. vét. Pays trop., 23: 301-307.

16. TAYLOR W.P., 1979. Protection of goats against "peste des petits ruminants" with attenuated tinderpest virus. Res. vet. Sci., 27: 321-324.

BIDJEH K., BORNAREL P., IMADINE M., LANCELOT R. First time isolation of the PPR virus in Chad and experimental induction of the disease. Revue Elev. Méd. vét. Pays trop., 1995, 48 (4) : 295-300

In Chad, in 1993-1994, investigations into "peste des petits ruminants" (PPR) outbreaks were carried out in flocks of Sahelian goats. Although in the carly 1993 outbreaks the virus had not becn isolated yet, a scrological prevalence $(34 \%)$ was observed using ELISA test in 475 sera collected in the infested area. The virus was then isolated for the first time in Chad and typical PPR cases were observed in Sahelian goats, known to be little sensitive to the virus. To experimentally induce the disease, goats were inoculated with suspensions of lymph nodes or lungs, collected from sick animals Lung suspensions induced the most serious symptoms. Suspensions of lungs and mesenteric lymph nodes were positive by agar-gel immunodiffusion test against PPR anti-serum, while prescapular lymph nodes remained negative. Characterization of the isolated virus, in particular with regard to its genomic identity, should be investigated.

Key words: Cattle - Goat - Morbillivirus - Experimental infection - Serology - ELISA - Immunology - Epidemiology - Chad. 